\title{
ARTICLE
}

Received 16 Jul 2012 | Accepted 19 Jul 2013 | Published 12 Aug 2013 DOI: 10.1038/ncomms3326

\section{In vivo time-gated fluorescence imaging with biodegradable luminescent porous silicon nanoparticles}

Luo Gu,2,3, David J. Hall ${ }^{4,5}$, Zhengtao Qin', Emily Anglin', Jinmyoung Joo1, David J. Mooney 2,3, Stephen B. Howell ${ }^{5,6} \&$ Michael J. Sailor ${ }^{1,5}$

Fluorescence imaging is one of the most versatile and widely used visualization methods in biomedical research. However, tissue autofluorescence is a major obstacle confounding interpretation of in vivo fluorescence images. The unusually long emission lifetime (5-13 $\mu \mathrm{s})$ of photoluminescent porous silicon nanoparticles can allow the time-gated imaging of tissues in vivo, completely eliminating shorter-lived ( $<10 \mathrm{~ns}$ ) emission signals from organic chromophores or tissue autofluorescence. Here using a conventional animal imaging system not optimized for such long-lived excited states, we demonstrate improvement of signal to background contrast ratio by $>50$-fold in vitro and by $>20$-fold in vivo when imaging porous silicon nanoparticles. Time-gated imaging of porous silicon nanoparticles accumulated in a human ovarian cancer xenograft following intravenous injection is demonstrated in a live mouse. The potential for multiplexing of images in the time domain by using separate porous silicon nanoparticles engineered with different excited state lifetimes is discussed.

\footnotetext{
${ }^{1}$ Department of Chemistry and Biochemistry, University of California, San Diego, La Jolla, California 92093, USA. ${ }^{2}$ School of Engineering and Applied Sciences, Harvard University, Cambridge, Massachusetts 02138, USA. ${ }^{3}$ Wyss Institute for Biologically Inspired Engineering, Harvard University, Cambridge, Massachusetts 02138, USA. ${ }^{4}$ Department of Radiology, University of California, San Diego, La Jolla, California 92093, USA. ${ }^{5}$ Moores Cancer Center, University of California, San Diego, La Jolla, California 92093, USA. ${ }^{6}$ Department of Medicine, University of California, San Diego, La Jolla, California 92093, USA. Correspondence and requests for materials should be addressed to M.J.S. (email: msailor@ucsd.edu).
} 
F luorescence imaging has become a dominant in vitro and in vivo visualization method in biomedical research due to its high sensitivity, its high spatial resolution and its ease of use $^{1,2}$. In vivo imaging of exogenous fluorescent probes that target diseased tissues has also shown promising results in clinical settings, such as the early detection of breast cancer, the outlining of tumour margins during surgery and endoscopic diagnosis of cancer micrometastasis ${ }^{1,3-5}$. However, the method is limited by tissue attenuation (scattering and absorption of the excitation or the emission light) and by tissue autofluorescence ${ }^{6,7}$. To minimize tissue attenuation effects, researchers have concentrated on nearinfrared (NIR) fluorophores that are excited and emit in the spectral window between wavelengths of $650-950 \mathrm{~nm}^{8-11}$. However, tissue autofluorescence still produces a substantial background signal in this spectral range that severely limits the quality of images, especially when very low concentrations of the fluorescent probe accumulate at the target site ${ }^{12}$.

The endogenous fluorophores responsible for tissue autofluorescence have decay lifetimes of $\sim 1-10 \mathrm{~ns}$, depending on the type of tissue ${ }^{7}$. It has been proposed that late time-gating (that is, capturing the signal at a delayed time after excitation) could be used to image molecular or quantum dot imaging probes in the presence of this interference ${ }^{3-18}$. However, there is a lack of biocompatible NIR fluorophores with fluorescence lifetimes significantly greater than $1-10 \mathrm{~ns}$, due to the quantum mechanical selection rules associated with organic molecules or direct gap semiconductors ${ }^{7,19}$.

Electrochemically etched porous silicon has shown considerable potential for in vivo applications due to its biodegradability, its low toxicity, its large specific capacity for drug loading and its intrinsic photoluminescence ${ }^{20-27}$. Silicon is an indirect gap semiconductor, which gives it a much longer-lived excited state than direct gap semiconductors such as $\mathrm{CdS}$ or $\mathrm{CdSe}^{28}$. This property translates to the scale of quantum dots, and nanoparticles derived from silicon tend to display radiative lifetimes on the order of $100 \mathrm{~ns}$ to several microseconds ${ }^{29-31}$. In contrast, CdS and CdSe excited states decay on much shorter timescales of a few nanoseconds to several tens of nanoseconds ${ }^{32}$.
We recently demonstrated that NIR luminescent nanoparticles made of porous silicon (LPSiNPs) can be prepared that are biocompatible, have low systemic toxicity, and that accumulate in tumours in vivo and then degrade into components cleared by the kidneys $^{33}$.

Here we show that the emission lifetime of these nanoparticles

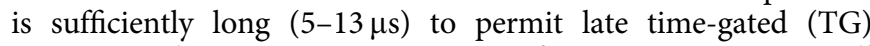
imaging. The persistent emission from LPSiNPs is well differentiated in the time domain from signals associated with tissue autofluorescence or interfering organic chromophores. Delayed (late TG) images acquired $18 \mathrm{~ns}$ after excitation show clear signals from LPSiNPs, while the background fluorescence has disappeared due to its much shorter lifetime. An example of the utility of the method is demonstrated in the imaging of a human ovarian cancer xenograft in a mouse model, using LPSiNP probes introduced via intravenous injection.

\section{Results}

Long emission lifetime of LPSiNPs. LPSiNPs were prepared by electrochemical etching of single-crystal $\mathrm{Si}$ in HF-containing electrolyte, followed by lifting-off of the porous layer, ultrasonic fracture, filtration of the resulting nanoparticles through a $0.22-\mu \mathrm{m}$ filter membrane, and finally activation of photoluminescence by treatment in an aqueous solution following the published procedure $^{33}$. The emission lifetime of the nanoparticles was controlled by adjusting the current density used in the electrochemical etch (Fig. 1a and Supplementary Figs S1,S2) and by post-etching chemical treatments (Supplementary Table S1). For the TG fluorescence imaging study presented here, the LPSiNPs were prepared using electrochemical etching at a current density of $200 \mathrm{~mA} \mathrm{~cm}^{-2}$ with a 2 -week aqueous activation treatment. The nanoparticles were coated with $5 \mathrm{kDa}$ polyethylene glycol (PEG) by reaction with a PEG-silane (PEG-LPSiNP, Fig. 1b). The mean hydrodynamic diameter of the PEG-LPSiNP formulation (measured by dynamic light scattering, DLS) was $168 \mathrm{~nm}$, consistent with transmission electron microscopy measurements (Supplementary Fig. S3). The photoluminescence spectrum a

\begin{tabular}{cccc}
\hline $\begin{array}{c}\text { Etching current } \\
\text { density }\left(\mathrm{mA} \mathrm{cm}^{-2}\right)\end{array}$ & $\begin{array}{c}\text { DLS size } \\
(\mathrm{nm})\end{array}$ & Surface & $\begin{array}{c}\text { Lifetime } \\
(\mu \mathrm{s})\end{array}$ \\
\hline 50 & 143 & Oxide & 5.1 \\
200 & 160 & Oxide & 10.0 \\
400 & 159 & Oxide & 12.1 \\
\hline
\end{tabular}

b

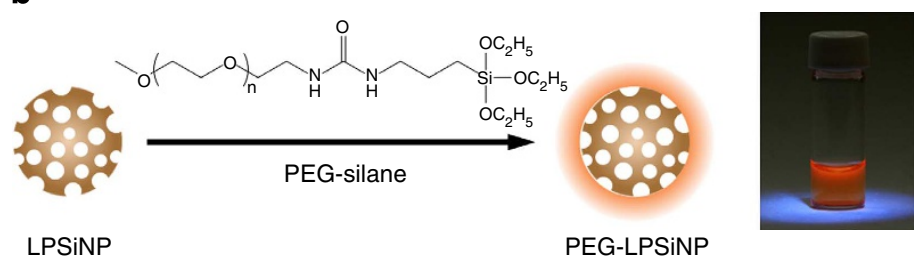

c

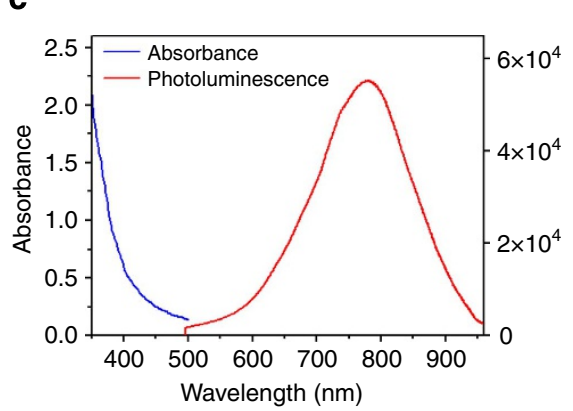

d

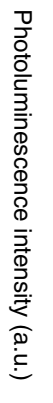

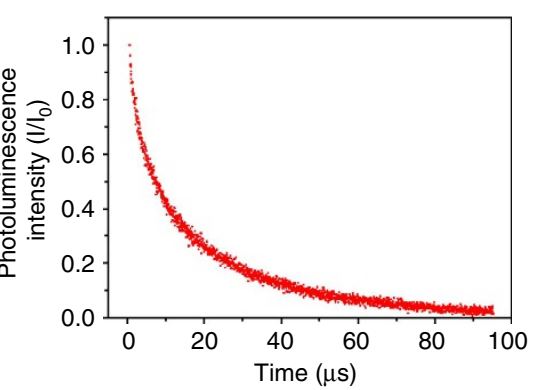

e

Figure 1 | Characterization of PEG-conjugated luminescent porous silicon nanoparticles. (a) Hydrodynamic diameter, surface chemistry and emission lifetime of LPSiNPs prepared at the indicated etch current densities. (b) Schematic diagram depicting the PEG surface chemistry on the LPSiNPs. Photograph of PEG-LPSiNPs in water, obtained under ultraviolet light illumination. (c) Absorbance and steady-state photoluminescence spectrum of PEGLPSiNPs $\left(\lambda_{\mathrm{ex}}=370 \mathrm{~nm}\right)$. (d) Normalized photoluminescence intensity-time trace for PEG-LPSiNPs after pulsed excitation $\left(\lambda_{\mathrm{ex}}=456 \mathrm{~nm}, \lambda_{\mathrm{em}}=650 \mathrm{~nm}\right.$, $22^{\circ} \mathrm{C}$ ). (e) Fluorescence lifetimes of commonly used fluorophores and tissue autofluorescence $\mathrm{e}^{7,42}$. 
from the PEG-LPSiNPs $\left(\lambda_{\mathrm{ex}}=370 \mathrm{~nm}\right)$ appeared at wavelengths between 600 and $900 \mathrm{~nm}$ (Fig. 1c), and the decay lifetime was $12 \mu \mathrm{s}$ $\left(\lambda_{\mathrm{em}}=650 \mathrm{~nm}, 22^{\circ} \mathrm{C}\right)$. This lifetime is $>1,000$ times larger than that typical of tissue autofluorescence or common fluorescent imaging dyes (Fig. 1d,e; Supplementary Figs S4,S5).

We first tested whether the long-lived photoluminescence from PEG-LPSiNPs could be separated from the fluorescence of conventional fluorophores in the time domain. To demonstrate the potential for practical applications, we used a commercial in vivo imaging system (eXplore Optix, ART Inc.). This instrument uses pulsed laser diodes and time-correlated single-photon counting to quantify fluorescence intensity and lifetime $^{34}$. When imaged using a 470-nm excitation laser and a 590-nm longpass emission filter, the intensity of fluorescence from an aqueous solution $10 \mu \mathrm{g} \mathrm{ml}^{-1}$ of the common imaging dye Cy3.5 was comparable to the intensity of photoluminescence from an aqueous suspension $100 \mu \mathrm{g} \mathrm{ml}^{-1}$ in PEGLPSiNPs (Fig. 2a). The laser we used operates at a repetition rate of $40 \mathrm{MHz}$, corresponding to a time window between pulses of $25 \mathrm{~ns}$. The duration of an individual pulse from the laser is $<0.1 \mathrm{~ns}$. Although the Cy3.5 sample and the PEG-LPSiNP sample showed similar photoluminescence intensity when integrated over the entire $25 \mathrm{~ns}$ window (Fig. 2a), temporally integrating a $1 \mathrm{~ns}$ gate of the signal between 20.5 and $21.5 \mathrm{~ns}$ of the imaging window (18 and 19 ns post-pulse after correcting for synchronization) yielded a strong signal from the PEG-LPSiNP sample (50 times greater than background), while the signal from the Cy3.5 sample was at or below the noise level of the instrument (Fig. 2b,c). This improvement in image contrast is due to the rapid decay of fluorescence from the organic fluorophore ( $0.6 \mathrm{~ns}$ lifetime); the photoluminescence signal from the nanoparticle is essentially constant for the duration of the $25 \mathrm{~ns}$ period between pulses (Fig. $2 \mathrm{~b}$ ). As a result, only the PEGLPSiNP sample was visible under late time-gating conditions (Fig. 2c).

In vivo TG imaging with PEG-LPSiNPs. We next investigated the possibility of eliminating tissue autofluorescence or interference from exogenous fluorophores when imaging PEGLPSiNPs in vivo. A $20-\mu \mathrm{l}$ aliquot of PEG-LPSiNPs $\left(0.5 \mathrm{mg} \mathrm{ml}^{-1}\right)$ and a $20-\mu \mathrm{l}$ aliquot of $\mathrm{Cy} 3.5\left(0.02 \mathrm{mg} \mathrm{ml}^{-1}\right)$ in normal saline a

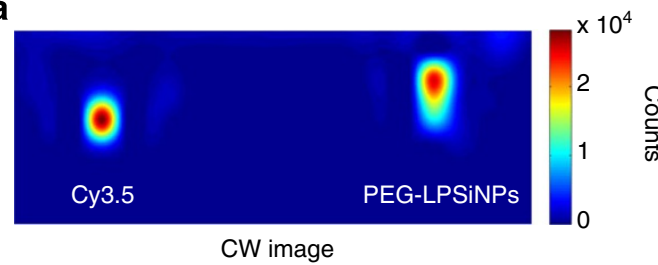

C

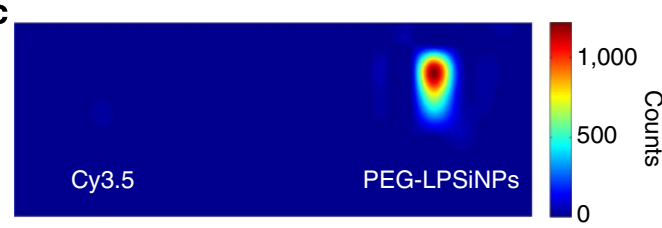

TG image b

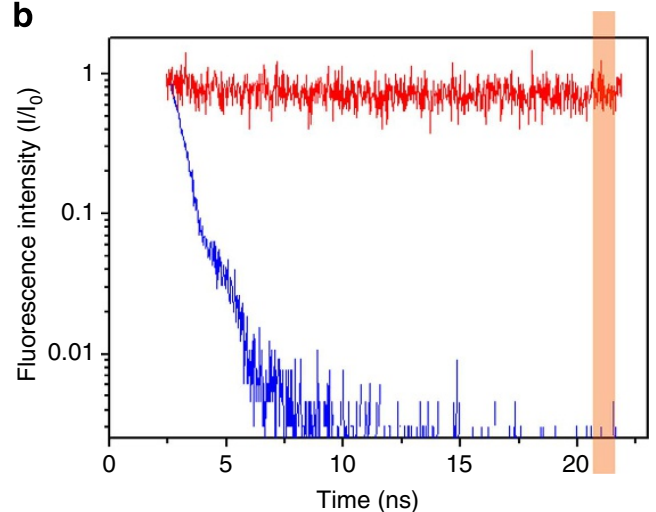

d

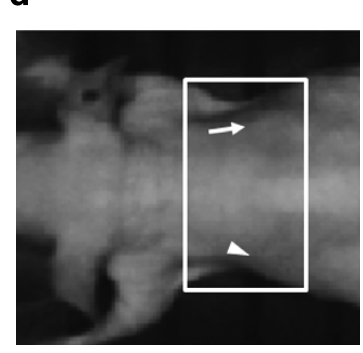

e

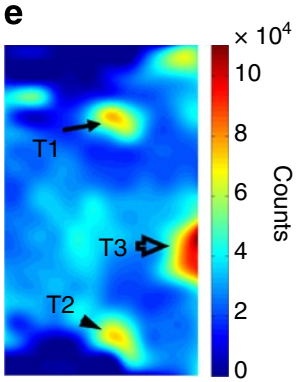

CW image f

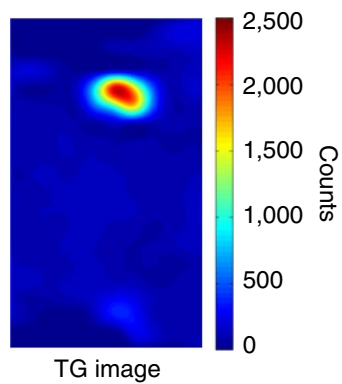

g

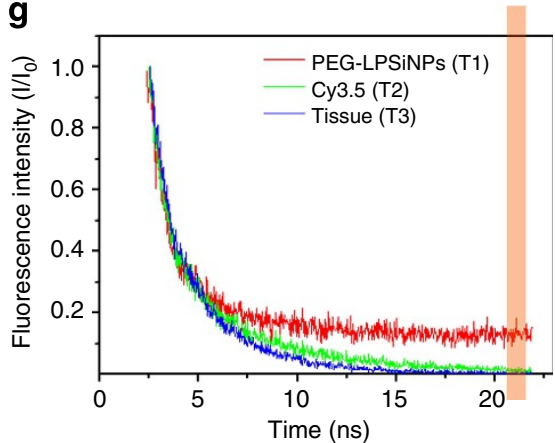

Figure 2 | Comparison of time-domain fluorescence characteristics of PEG-LPSiNPs and the common imaging fluorophore Cy3.5. (a) Steady-state, or CW fluorescence images of Cy3.5 $\left(0.01 \mathrm{mg} \mathrm{ml}^{-1}\right)$ and PEG-LPSiNPs $\left(0.1 \mathrm{mg} \mathrm{ml}^{-1}\right)$ samples in microtubes $\left(\lambda_{\mathrm{ex}}=470 \mathrm{~nm}, \lambda_{\mathrm{em}}=590 \mathrm{~nm}\right.$ longpass $)$. (b) Normalized intensity decay of the fluorescence/photoluminescence signals from the Cy3.5 and PEG-LPSiNPs samples shown in (a) as a function of time after excitation pulse. The vertical orange bar depicts the time window used in the time-gating algorithm (20.5-21.5 ns of the $25 \mathrm{~ns}$ imaging window, which is $18-19 \mathrm{~ns}$ after excitation pulse) to obtain TG fluorescence images; that is, the images depicted as 'TG' represent fluorescence intensity integrated between 18-19 ns after the excitation pulse. (c) TG image of the same microtubes in (a). The image of the Cy3.5 sample almost completely disappears due to its short-lived emission. (d) Bright field image of a nude mouse injected subcutaneously with PEG-LPSiNPs $\left(20 \mu l, 0.5 \mathrm{mg} \mathrm{ml}^{-1}\right)$ and Cy3.5 $(20 \mu \mathrm{l}$, $0.02 \mathrm{mg} \mathrm{ml}^{-1}$ ). The arrow indicates the injection site of PEG-LPSiNPs, and the arrowhead indicates the injection site of Cy3.5. (e) CW fluorescence image of the region of the mouse indicated by the white box in (d), obtained with the Optix imaging system. Regions identified as T1, T2 and T3 are the injection site of PEG-LPSiNPs, the injection site of Cy3.5, and the abdomen, respectively. (f) TG image of the same region shown in (e). Signals from the Cy3.5 dye (T2) and from tissue autofluorescence (T3) almost disappear. The intensity ratio of PEG-LPSiNPs to tissue autofluorescence was calculated by comparing the CW with the TG signal intensities of PEG-LPSiNPs (T1, arrow) and tissue autofluorescence (T3, hollow arrow) as indicated in (e).

(g) Normalized intensity decay of the fluorescence signals from T1, T2 and T3 in (e), as indicated. The vertical orange bar depicts the time window used in the time-gating algorithm for (f). 
were injected subcutaneously into the right and left shoulder of a nude mouse, respectively (Fig. 2d). When imaged under pseudo continuous wave $(\mathrm{CW})$, or steady-state conditions (that is, no time gating), the two injection points had intensities comparable to the brighter autofluorescent tissues (Fig. 2e). However, the intensity-time decay curves of the relevant regions revealed a distinct persistence of signal only for the PEG-LPSiNPs (Fig. $2 \mathrm{~g}$ ). As shown in Fig $2 \mathrm{~g}$, the fast decay component (dominant at times $<5 \mathrm{ns)}$ from ubiquitous tissue autofluorescence appeared similar in all the body regions imaged: the PEG-LPSiNP injection site (T1), the Cy3.5 injection site (T2) and the background tissue region (T3). Only at the site of PEG-LPSiNP injection was residual photoluminescence intensity observed at times $>5 \mathrm{~ns}$, consistent with the longer lifetime of PEG-LPSiNPs. As predicted by the in vitro data of Fig. 2b, the signal from PEG-LPSiNPs was relatively constant between $\sim 10$ and $22 \mathrm{~ns}$. To confirm this persistent signal at the injection site arose from PEG-LPSiNPs, we measured the full decay in vivo on a $100-\mu$ s timescale (Supplementary Fig. S5). In agreement with the results in Fig. 2g, both control tissue and tissue injected with PEG-LPSiNPs showed a prompt decay from tissue autofluorescence $(<5 \mathrm{~ns})$. However, only the tissue containing PEG-LPSiNPs showed a slow photoluminescence decay component, consistent with the in vitro measurements of PEG-LPSiNPs (Fig. 1d, Supplementary Fig. S5). Due to the slower radiative decay rate and low injection dose, this long-lived signal is weaker than the signals from Cy3.5 or from tissue autofluorescence, though it is easy to discern in the timeresolved data (Fig. 2g, T1). Application of the late time-gating algorithm (1 ns gate, $18 \mathrm{~ns}$ post-excitation) revealed a distinct spot in the PEG-LPSiNP injection point (T1), with negligible intensity from the Cy3.5 injection or from background tissue autofluorescence (Fig. 2f). Simple analysis of pixel intensities revealed a 20 -fold increase in image contrast with the TG image relative to the CW image (Fig. 2e and f). It is possible that excitation crossover (bleed-through of the excitation source scattered from the tissues), a common problem for in vivo fluorescence imaging, may also contribute to the background signals in CW fluorescence images. As the pulse width of the excitation laser was
$<0.1 \mathrm{~ns}$, the time-gating method used here was able to remove this potential interference.

PEG-LPSiNPs are distinguishable from fluorescent proteins. Genetically expressed fluorophores such as fluorescent proteins are widely used in biomedical research, so we next tested TG imaging of PEG-LPSiNPs in the human ovarian carcinoma 2008 cell line expressing the red fluorescent protein mCherry (2008mCherry, Supplementary Fig. S6a). The fluorescence lifetime of mCherry is $\sim 1.4 \mathrm{~ns}^{35}$, (verified in Supplementary Fig. S6b). In vitro imaging of PEG-LPSiNPs and 2008-mCherry cells showed that the signal from the nanoparticles was readily distinguishable using late TG imaging (Supplementary Fig. S6,c,d).

The PEG-LPSiNPs were also readily distinguishable from mCherry in vivo. A nude mouse bearing a 2008-mCherry tumour xenograft on each shoulder was imaged. The CW image displayed fluorescence from both tumours as well as strong tissue autofluorescence (Fig. 3a,b). The fluorescence signal from mCherry could not be distinguished from tissue autofluorescence due to their similar short fluorescence lifetimes (Fig. 3b). PEGLPSiNPs $\left(50 \mu \mathrm{l}, 0.2 \mathrm{mg} \mathrm{ml}^{-1}\right)$ were then injected into the tumour on the right shoulder of the mouse, and the mouse was imaged again under CW conditions. The tumour injected with nanoparticles was brighter than the control tumour in the CW image, but tissue autofluorescence and the signal from the control tumour were still clearly visible (Fig. 3c). In contrast, signals from both the control tumour and tissue autofluorescence were completely eliminated in the TG image (Fig. 3c). Ex vivo fluorescence images confirmed the presence of PEG-LPSiNPs in the tumour, and they demonstrated the effectiveness of TG imaging of PEG-LPSiNPs in ex vivo tissues (Fig. 3d-f).

TG imaging of tumours with PEG-LPSiNPs. Finally, we evaluated the potential of imaging tumours using intravenously injected circulating PEG-LPSiNPs as the photoluminescent probe. Intravenously injected nanoparticles can passively accumulate in tumour tissues due to the enhanced permeability and retention a

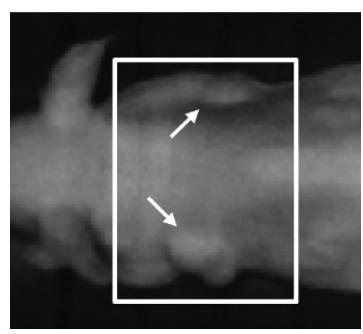

b

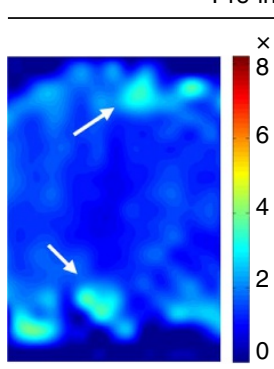

CW image
Pre-injection

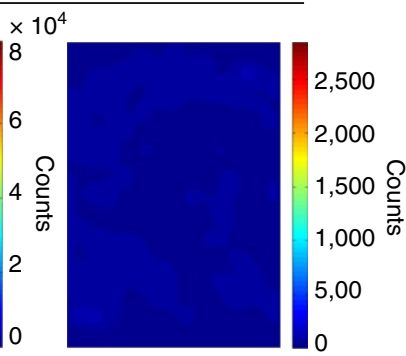

TG image

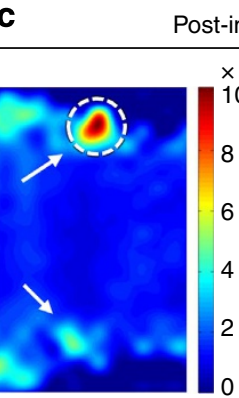

CW image
Post-injection

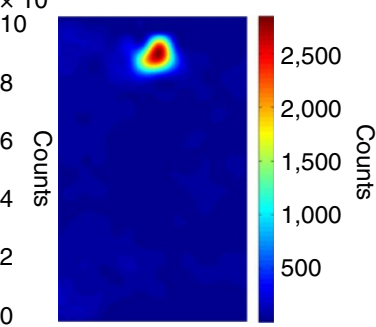

TG image

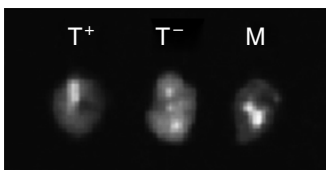

e

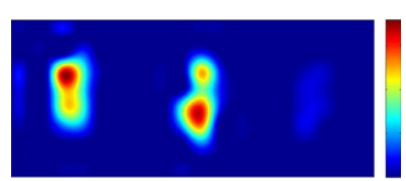

CW image f

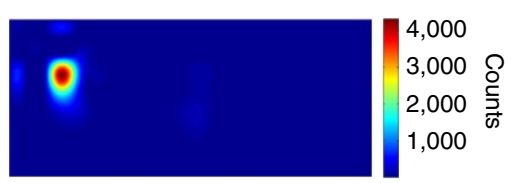

TG image

Figure 3 | TG fluorescence images comparing PEG-LPSiNP with fluorescent protein mCherry in a nude mouse bearing mCherry-expressing tumours. (a) Bright field photograph of mouse containing two tumours, one on each shoulder. Tumours are indicated with white arrows. (b) CW and TG (as defined in Fig. 2) fluorescence images of the tumours, showing low differentiation of the expressed mCherry and tissue autofluorescence in the time-domain. (c) CW and TG fluorescence images of the tumors after injection of PEG-LPSiNPs $\left(50 \mu \mathrm{l}, 0.2 \mathrm{mg} \mathrm{ml}^{-1}\right.$ ) into the right shoulder tumour (on the top of the image, indicated with dashed white circle). (d) Ex vivo bright field image of the PEG-LPSiNP-injected tumor $\left(\mathrm{T}^{+}\right)$, a control tumor $\left(\mathrm{T}^{-}\right.$) and muscle tissue excised from the animal post-injection. (e) CW fluorescence image of the excised tissues in (d). (f) TG fluorescence image of the tissues in (d). 
effect $^{36-38}$. However, the efficiency of this process is low and generally only a small fraction of injected nanoparticles accumulates in a tumour ${ }^{39,40}$. This creates a challenge for in vivo optical imaging, because tissue autofluorescence can overwhelm the signals from the fluorescent probe. To evaluate the ability of late TG PEG-LPSiNPs to remove tissue autofluorescence in this situation, PEG-LPSiNPs $\left(10 \mathrm{mg} \mathrm{kg}^{-1}\right)$ were injected intravenously into a nude mouse bearing a human ovarian carcinoma SKOV3 xenograft tumour (Fig. 4a). Fluorescent images of the mouse were intermittently acquired over a 24 -h time period post-injection. No signal from the nanoparticles was observed in either the CW or the TG images immediately after injection, because very few nanoparticles had accumulated in the tumour tissue (Fig. 4b). In addition, the lifetime of autofluorescence measured from the xenograft tumour was $2.2 \mathrm{~ns}$, similar to the lifetime of the autofluorescence from healthy tissues measured in the vicinity of the tumour $(2.3 \mathrm{~ns})$. Therefore, no tumour contrast was detected in the TG image if the long-lived photoluminescent PEG-LPSiNP probe was not present. Weak photoluminescence was detected in the tumour $1 \mathrm{~h}$ after injection. However, the signal from the nanoparticles was too weak to be differentiated from tissue autofluorescence even with time-gating (Fig. 4c). As time progressed more PEG-LPSiNPs accumulated at the tumour, and the TG image obtained $4 \mathrm{~h}$ post-injection clearly revealed the site of the tumour (Fig. 4d). No contrast between tumour and normal tissue was observed in the $\mathrm{CW}$ image due to the pronounced autofluorescence signals. The intensity ratio between the tumour and normal tissues increased from $\sim 1$ in the $\mathrm{CW}$ image to 3 in the TD image (Fig. 4d). The signal from the tumour then decreased substantially $24 \mathrm{~h}$ post-injection (Fig. $4 \mathrm{e}$ ) as the nanoparticles degraded and cleared from the host ${ }^{33}$. Ex vivo fluorescence images indicated that a small, but detectable quantity of PEG-LPSiNPs remained in the tumour $24 \mathrm{~h}$ postinjection (Supplementary Fig. S7).

\section{Discussion}

Although the commercial time-domain imager used in the present study to identify and track the silicon-based nanoparticles has very good (sub-nanosecond) time resolution and excellent detector sensitivity, it is limited in the delay time that can be applied due to the repetition rate of the pulsed laser. A less sophisticated imaging system, with an ability to gate at a modest ( $>50 \mathrm{~ns}$ or even longer) delay time, is expected to yield even better image quality from PEG-LPSiNPs due to the long (microseconds) emission lifetime of this probe (Fig. 1d, Supplementary Figs S4,S5). For example, given two emitters with the same steady-state emission intensity-a long-lived emitter such as porous $\mathrm{Si}$ (12 $\mu$ s lifetime, 10\% quantum yield) and a prompt-emitting fluorophore such as Cy3.5 (0.6 ns lifetime, 15\% quantum yield)-the TG (1 ns window, delay of $18 \mathrm{~ns}$ ) emission intensity of the porous $\mathrm{Si}$ emitter is theoretically $>10^{8}$ times larger than the prompt emitter. This calculation assumes singleexponential decays and unitary instrument response functions. The theoretical contrast ratio rises very quickly with delay time; if imaged at a delay of $50 \mathrm{~ns}$, it is $>10^{31}$. Even if the porous Si probe is present at a concentration a million fold lower than the prompt-emitting fluorophores, the theoretical contrast ratio at $50 \mathrm{~ns}$ post-excitation is $>10^{25}$. The potential sensitivity improvement obtained with later time-gating is especially helpful when a low dose of imaging probe is required.

Typically in time-resolved fluorescence imaging one must contend with temporally overlapping fluorescence decays from different fluorophores and unmix these signals based on lifetime contrast using model fitting (for example, multiexponential fits). However, late TG imaging using long-lifetime LPSiNPs avoids the temporal overlap of confounding tissue autofluorescence signals, eliminating the need to measure the complete fluorescence decay and unmix the signals. This approach provides a means of improving detection sensitivity and image contrast without a

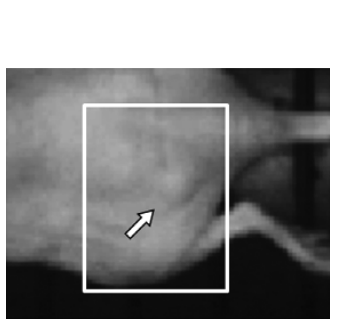

b

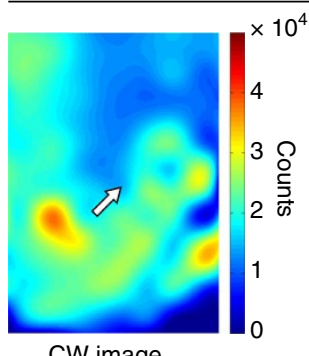

CW image
$0 \mathrm{~h}$ post-injection

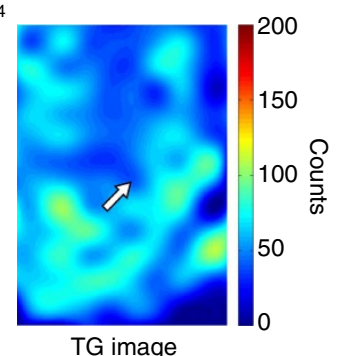

TG image c

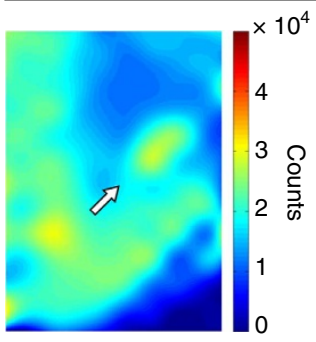

CW image
$1 \mathrm{~h}$ post-injection

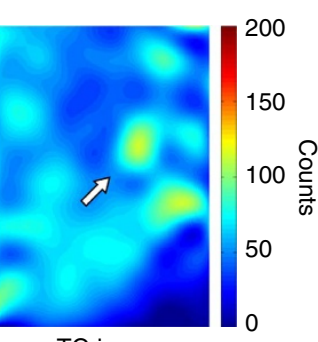

TG image d

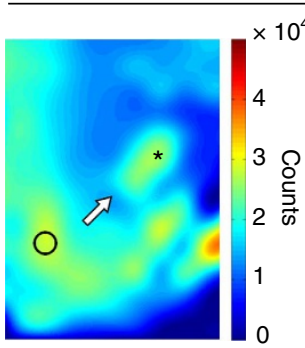

CW image
$4 \mathrm{~h}$ post-injection

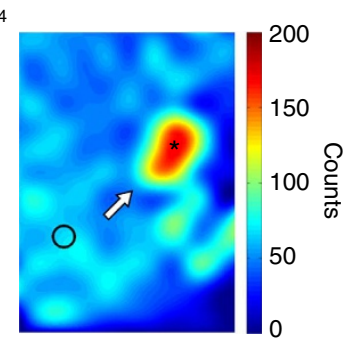

TG image e $24 \mathrm{~h}$ post-injection

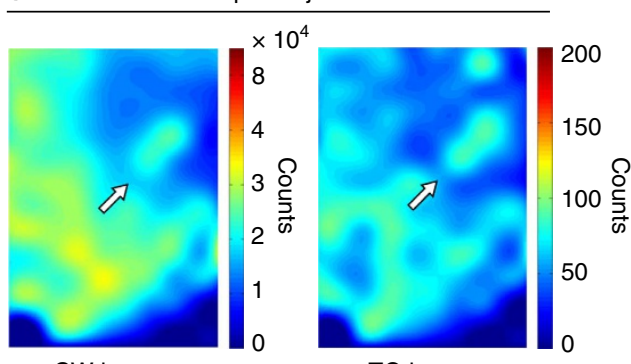

CW image

TG image

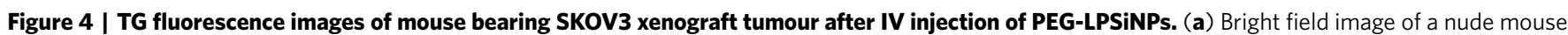
bearing a tumour at the flank. The arrow indicates the site of the tumour. (b-e) CW and TG (as defined in Fig. 2) fluorescence images of the region indicated with the white box in (a) immediately (b), $1 \mathrm{~h}(\mathbf{c}), 4 \mathrm{~h}$ (d) or $24 \mathrm{~h}$ (e) post-injection of PEG-LPSiNPs (10 mg kg ${ }^{-1}$ body weight). The signal to background (tissue autofluorescence) ratio described in the text was calculated by comparing signal intensities at the sites indicated by the black asterisk (PEG-LPSiNPs) and the black circle (tissue autofluorescence) as indicated in (d) in both CW and TG images. 
sacrificing image resolution or generating false readings due to potential errors in model assumptions.

The wavelength distribution of the NIR emission spectrum of LPSiNPs is broader than many fluorescent probes, which places a limit on the use of LPSiNPs for multicolour imaging in the spectral domain. However, in contrast to conventional molecular probes, the decay lifetime of LPSiNPs can be readily tuned without changing the chemical nature of the probe, using a mild aqueous treatment (Supplementary Table S1). This provides the possibility of multiplex imaging in the time domain using multiple nanoparticle probes and various time gates. Furthermore, the slow change in emission lifetime that occurs upon degradation in aqueous media may allow the nanoparticle probe to report on its age in the system. In addition to their ability to provide high contrast images by time gating, the low toxicity and biodegradable characteristics of LPSiNPs overcomes the environmental and safety disadvantages of cytotoxic or non-biodegradable fluorescent probes currently used for in vitro or in vivo imaging.

\section{Methods}

Preparation of PEG-LPSiNPs. Luminescent porous silicon nanoparticles (LPSiNPs) were prepared following a previously described method ${ }^{33,41}$ : (100)-oriented p-type single-crystal Si wafers $(0.8-1.1 \mathrm{~m} \Omega$-cm resistivity, obtained from Siltronix, Inc.) were electrochemically etched by application of a constant current density of $200 \mathrm{~mA} \mathrm{~cm}^{-2}$ for $150 \mathrm{~s}$ in an electrolyte containing aqueous $48 \%$ hydrofluoric acid and ethanol in a 3:1 ratio. The resulting porous Si films were lifted from the Si substrate by application of a current pulse of $4 \mathrm{~mA} \mathrm{~cm}^{-2}$ for $250 \mathrm{~s}$ in a solution containing $3.3 \%$ (by volume) of $48 \%$ aqueous HF in ethanol. The porous Si film was fractured by ultrasound and filtered through a $0.22-\mu \mathrm{m}$ membrane. Finally, the nanoparticles were mildly oxidized by soaking in deionized water for 2 weeks to activate photoluminescence. The activated nanoparticles were rinsed with deionized water three times by centrifugation. LPSiNP samples were prepared at the following etching current densities: $50 \mathrm{~mA} \mathrm{~cm}^{-2}$ for $300 \mathrm{~s}$, $200 \mathrm{~mA} \mathrm{~cm}^{-2}$ for $150 \mathrm{~s}$ or $400 \mathrm{~mA} \mathrm{~cm}^{-2}$ for $150 \mathrm{~s}$. The activation step involved soaking of the LPSiNPs in deionized water for various periods of time: 2, 6 or 10 weeks. To prepare PEG-LPSiNPs, a $0.6-\mathrm{ml}$ aliquot of an ethanolic dispersion $\left(0.5 \mathrm{mg} \mathrm{ml}^{-1}\right.$ LPSiNPs) was mixed with a $0.5-\mathrm{ml}$ aliquot of $6 \mathrm{mg} \mathrm{ml}^{-1} \mathrm{mPEG}$ Silane (MW $5 \mathrm{k}$, Laysan Bio, Inc.) in ethanol. The mixture was stirred for $16 \mathrm{~h}$ at room temperature. The nanoparticles were rinsed three times with ethanol and then three times with water. The samples were collected by centrifugation between each rinsing step.

Property measurements of LPSiNPs. Dynamic light scattering (Zetasizer Nano ZS90, Malvern Instruments) was used to determine the hydrodynamic size of the nanoparticles. Transmission electron microscope images were obtained with a FEI Tecnai $G^{2}$ Sphera. Scanning electron microscope images were obtained using a Philips XL30 field emission ESEM operating in secondary electron emission mode. The photoluminescence ( $\lambda_{\text {ex }}=370$ and $460 \mathrm{~nm}$ long pass emission filter) spectra of LPSiNPs or PEG-LPSiNPs were obtained using a Princeton Instruments/Acton spectrometer fitted with a liquid nitrogen-cooled silicon charge-coupled device (CCD) detector. The photoluminescence decay data for aqueous dispersions of nanoparticles (in water or in tissue homogenates) were acquired using a Horiba Scientific FluoroLog-3 spectrofluorometer using a time-correlated single-photon counting method. A 456-nm NanoLED (Horiba Scientific) at $10 \mathrm{kHz}$ repetition rate was used as the excitation source. The signal was collected at 650 or $700 \mathrm{~nm}$ at $22^{\circ} \mathrm{C}$. Although the decay curves for the PSiNPs were nonexponential, average decay lifetime is reported as the time at which the photoluminescence intensity of the nanoparticles decreased to $1 / e$ of the initial value after excitation. For the in vivo photoluminescence decays obtained on a longer (microseconds) timescale (Supplementary Fig. S5), spectra were acquired using a home-built system. A $10-\mathrm{Hz}$ pulsed Nd:YAG laser (excitation at $355 \mathrm{~nm}$, Quantel) was used as the source, and the PL signal was recorded with a high speed Si photo detector (DET110, Thorlabs) coupled to a 400-nm longpass filter and an oscilloscope (DPO3054, Tektronix).

Cell lines and mice. Two human ovarian carcinoma cell lines SKOV3 and 2008 were used in this study. In all, 2008 cells were transduced with lentiviral vectors containing the red fluorescent protein mCherry sequence (pLVX-mCherry, Clontech). The cells expressing mCherry (2008-mCherry) were sorted by flow cytometry and maintained in RPMI-1640 medium with $10 \%$ fetal calf serum (Invitrogen). Female nu/nu nude mice (Charles River) were maintained in specific pathogen-free facilities at the University of California, San Diego. Animal protocols were approved by the Institutional Animal Care and Use Committee.
In vitro and in vivo fluorescence imaging. A time-domain fluorescence imaging system eXplore Optix (ART Advanced Research Technologies, Inc.) was used to image fluorophore solutions and cell suspensions in vitro, and mice in vivo. A $470-\mathrm{nm}$ laser with $40 \mathrm{MHz}$ repetition rate was used as the excitation source and a 590-nm longpass filter was used as the emission filter for all imaging experiments. For in vitro comparison of PEG-LPSiNPs and Cy3.5, an aliquot of PEG LPSiNPs in aqueous dispersion $\left(50 \mu \mathrm{l}, 0.1 \mathrm{mg} \mathrm{ml}^{-1}\right)$ was placed in a microtube, and an aliquot of Cy3.5 NHS ester (GE Healthcare) in aqueous solution ( $50 \mu \mathrm{l}$, $0.01 \mathrm{mg} \mathrm{ml}^{-1}$ ) was placed in a separate microtube. The microtubes were placed next to each other and imaged at the same time with the Optix imaging system. For in vivo imaging, PEG-LPSiNPs $\left(20 \mu \mathrm{l}, 0.5 \mathrm{mg} \mathrm{ml}^{-1}\right)$ and Cy3.5 $(20 \mu \mathrm{l}$,

$0.02 \mathrm{mg} \mathrm{ml}^{-1}$ ) were injected subcutaneously into the right and left shoulder of a nude mouse, respectively. The mouse was imaged with the Optix imaging system immediately after injection.

For in vitro comparison of PEG-LPSiNPs with mCherry, aliquots of PEG-LPSiNPs $\left(20 \mu \mathrm{l}, 0.2 \mathrm{mg} \mathrm{ml}^{-1}\right)$ and 2008 -mCherry cell suspension $(20 \mu \mathrm{l}$, $\sim 1$ million cells) were placed in separate microtubes and imaged at the same time with the Optix imaging system. For in vivo imaging, a nude mouse bearing a 2008-mCherry tumour $(\sim 0.7 \mathrm{~cm})$ on each side of the shoulder was used. An aliquot of PEG-LPSiNPs dispersion $\left(50 \mu \mathrm{l}, 0.2 \mathrm{mg} \mathrm{ml}^{-1}\right)$ was injected into the tumour on the right shoulder of the mouse, and the mouse was imaged with the Optix imaging system immediately after injection.

Nude mice bearing a SKOV3 tumour $(\sim 0.5 \mathrm{~cm}$, left flank) were used for the in vivo tumour imaging study. A PEG-LPSiNPs saline dispersion was injected intravenously into the mouse at a dose of $10 \mathrm{mg} \mathrm{kg}^{-1}$ body mass through the tail vein. The mouse was imaged at several different time points for $24 \mathrm{~h}$ post-injection. The tumour and muscle in the vicinity of the tumour were harvested and imaged $24 \mathrm{~h}$ after injection.

Data analysis and time gating. The Optix imaging system data were analysed using OptiView (ART Advanced Research Technologies, Inc.) to calculate the in vivo decay lifetimes of the fluorophores or tissue autofluorescence. CW fluorescence images were obtained by reconstructing the photoluminescent signal collected within the full $25 \mathrm{~ns}$ imaging time-window from the data using MATLAB (MathWorks, Inc.). TG fluorescence images were generated by extracting and temporally integrating the photoluminescent signal collected between 20.5 and $21.5 \mathrm{~ns}$ of the imaging time-window (18-19 ns after the excitation pulse) from the data using a subroutine in the computer program MATLAB.

\section{References}

1. Ntziachristos, V. Fluorescence molecular imaging. Annu. Rev. Biomed. Eng. 8, 1-33 (2006).

2. Leblond, F., Davis, S. C., Valdes, P. A. \& Pogue, B. W. Pre-clinical whole-body fluorescence imaging: review of instruments, methods and applications. J. Photochem. Photobiol. B Biol. 98, 77-94 (2010).

3. Corlu, A. et al. Three-dimensional in vivo fluorescence diffuse optical tomography of breast cancer in humans. Opt. Exp. 15, 6696-6716 (2007).

4. Nguyen, Q. T. et al. Surgery with molecular fluorescence imaging using activatable cell-penetrating peptides decreases residual cancer and improves survival. Proc. Natl Acad. Sci. USA 107, 4317-4322 (2010).

5. Kelly, K., Alencar, H., Funovics, M., Mahmood, U. \& Weissleder, R. Detection of invasive colon cancer using a novel, targeted, library-derived fluorescent peptide. Cancer Res. 64, 6247-6251 (2004).

6. Richards-Kortum, R. \& Sevick-Muraca, E. Quantitative optical spectroscopy for tissue diagnosis. Annu. Rev. Phys. Chem. 47, 555-606 (1996).

7. Berezin, M. Y. \& Achilefu, S. Fluorescence lifetime measurements and biological imaging. Chem. Rev. 110, 2641-2684 (2010).

8. Weissleder, R. A clearer vision for in vivo imaging. Nat. Biotechnol. 19, 316-317 (2001).

9. Bashkatov, A. N., Genina, E. A., Kochubey, V. I. \& Tuchin, V. V. Optical properties of human skin, subcutaneous and mucous tissues in the wavelength range from 400-2000 nm. J. Phys. D Appl. Phys. 38, 2543-2555 (2005).

10. Hilderbrand, S. A. \& Weissleder, R. Near-infrared fluorescence: application to in vivo molecular imaging. Curr. Opin. Chem. Biol. 14, 71-79 (2010).

11. Kobayashi, H., Ogawa, M., Alford, R., Choyke, P. L. \& Urano, Y. New strategies for fluorescent probe design in medical diagnostic imaging. Chem. Rev. 110, 2620-2640 (2010).

12. Gao, X. H., Cui, Y. Y., Levenson, R. M., Chung, L. W. K. \& Nie, S. M. In vivo cancer targeting and imaging with semiconductor quantum dots. Nat. Biotechnol. 22, 969-976 (2004)

13. Cubeddu, R., Canti, G., Taroni, P. \& Valentini, G. Time-gated fluorescence imaging for the diagnosis of tumours in a murine model. Photochem. Photobiol. 57, 480-485 (1993)

14. Dahan, M. et al. Time-gated biological imaging by use of colloidal quantum dots. Opt. Lett. 26, 825-827 (2001).

15. Hall, D. J., Sunar, U., Farshchi-Heydari, S. \& Han, S.-H. In vivo simultaneous monitoring of two fluorophores with lifetime contrast using a full-field time domain system. Appl. Opt. 48, D74-D78 (2009). 
16. Erten, A. et al. Enhancing magnetic resonance imaging tumour detection with fluorescence intensity and lifetime imaging. J. Biomed. Opt 15, 066012 (2010).

17. Hall, D. J. et al. Dynamic optical imaging of metabolic and NADPH oxidasederived superoxide in live mouse brain using fluorescence lifetime unmixing. J. Cereb. Blood Flow Metab. 32, 23-32 (2012).

18. Kumar, A. T. N. et al. Feasibility of in vivo imaging of fluorescent proteins using lifetime contrast. Opt. Lett. 34, 2066-2068 (2009).

19. Berezin, M. Y. et al. Long fluorescence lifetime molecular probes based on near infrared pyrrolopyrrole cyanine fluorophores for in vivo imaging. Biophys. J. 97, L22-L24 (2009).

20. Anderson, S. H. C., Elliott, H., Wallis, D. J., Canham, L. T. \& Powell, J. J. Dissolution of different forms of partially porous silicon wafers under simulated physiological conditions. Physica Status Solidi A Appl. Res. 197, 331-335 (2003).

21. Low, S. P., Voelcker, N. H., Canham, L. T. \& Williams, K. A. The biocompatibility of porous silicon in tissues of the eye. Biomaterials 30, 2873-2880 (2009).

22. Salonen, J., Kaukonen, A. M., Hirvonen, J. \& Lehto, V. P. Mesoporous silicon in drug delivery applications. J. Pharm. Sci. 97, 632-653 (2008).

23. Tasciotti, E. et al. Mesoporous silicon particles as a multistage delivery system for imaging and therapeutic applications. Nat. Nanotechnol. 3, 151-157 (2008).

24. Gu, L., Park, J.-H., Duong, K. H., Ruoslahti, E. \& Sailor, M. J. Magnetic luminescent porous silicon microparticles for localized delivery of molecular drug payloads. Small 6, 2546-2552 (2010).

25. Gelloz, B., Kojima, A. \& Koshida, N. Highly efficient and stable luminescence of nanocrystalline porous silicon treated by high-pressure water vapor annealing. Appl. Phys. Lett. 87, 031107 (2005).

26. Anglin, E. J., Cheng, L., Freeman, W. R. \& Sailor, M. J. Porous silicon in drug delivery devices and materials. Adv. Drug. Deliv. Rev. 60, 1266-1277 (2008)

27. Cheng, L. et al. Intravitreal properties of porous silicon photonic crystals: a potential self-reporting intraocular drugdelivery vehicle. Br. J. Ophthalmol. 92, 705-711 (2008)

28. Sze, S. M. Physics of Semiconductor Devices (John Wiley \& Sons, New York, 1981).

29. von Behren, J., van Buuren, T., Zacharias, M., Chimowitz, E. H. \& Fauchet, P. M. Quantum confinement in nanoscale silicon: the correlation of size with bandgap and luminescence. Solid State Commun. 105, 317-322 (1998).

30. Cullis, A. G., Canham, L. T. \& Calcott, P. D. J. The structural and luminescence properties of porous silicon. J. Appl. Phys. 82, 909-965 (1997).

31. Sailor, M. J. \& Wu, E. C. Photoluminescence-based sensing with porous silicon films, microparticles, and nanoparticles. Adv. Function. Mater. 19, 3195-3208 (2009).

32. Fisher, B., Caruge, J. M., Zehnder, D. \& Bawendi, M. Room-temperature ordered photon emission from multiexciton states in single CdSe core-shell nanocrystals. Phys. Rev. Lett. 94, 087403 (2005).

33. Park, J.-H. et al. Biodegradable luminescent porous silicon nanoparticles for in vivo applications. Nat. Mater. 8, 331-336 (2009).

34. Gallant, P. et al.Biomedical Topical Meeting, Vol. OSA Technical Digest (Optical Society of America), paper WD2 (2004).

35. Merzlyak, E. M. et al. Bright monomeric red fluorescent protein with an extended fluorescence lifetime. Nat. Methods 4, 555-557 (2007).
36. Iyer, A. K., Khaled, G., Fang, J. \& Maeda, H. Exploiting the enhanced permeability and retention effect for tumour targeting. Drug Discov. Today. 11, 812-818 (2006).

37. Peer, D. et al. Nanocarriers as an emerging platform for cancer therapy. Nat. Nanotechnol. 2, 751-760 (2007).

38. Brigger, I., Dubernet, C. \& Couvreur, P. Nanoparticles in cancer therapy and diagnosis. Adv. Drug. Deliv. Rev. 54, 631-651 (2002).

39. Ruoslahti, E., Bhatia, S. N. \& Sailor, M. J. Targeting of drugs and nanoparticles to tumours. J. Cell Biol. 188, 759-768 (2010).

40. Minchinton, A. I. \& Tannock, I. F. Drug penetration in solid tumours. Nat. Rev Cancer 6, 583-592 (2006).

41. Sailor, M. J. Porous Silicon in Practice: Preparation, Characterization, and Applications (Wiley-VCH, Weinheim, Germany, 2012).

42. Buschmann, V., Weston, K. D. \& Sauer, M. Spectroscopic study and evaluation of red-absorbing fluorescent dyes. Bioconjug. Chem. 14, 195-204 (2003).

\section{Acknowledgements}

We thank Professor Akif Tezcan and Professor Adah Almutairi for use of the fluorescence spectrometers and the UCSD IVISR facility for use of the eXplore Optix imaging system. We also gratefully acknowledge Dr Matheiu Lessard-Viger and Maria Angelella for their support with acquiring the photoluminescence lifetime measurements This work was supported by Grants U54-CA 119335, 5-R01-CA124427, CA095298, CA152185, 1DP20D006499-01, P50CA128346 (D.J.H.) and 5R01EB015498 (D.J.M.) from the National Institutes of Health, by DARPA under Cooperative Agreement HR0011-13-2-0017, and by grant DMR-1210417 from the National Science Foundation. L.G. was partially supported by the UCSD HHMI/NIBIB Interfaces Graduate Training Program (HHMI Grant HHMI56005681). This work was performed in part at Harvard University Center for Nanoscale Systems (CNS), a member of the National Nanotechnology Infrastructure Network (NNIN), which is supported by the National Science Foundation under NSF award no. ECS-0335765. We thank Dr Arthur McClelland at CNS for assistance with some of the in vivo photoluminescence decay experiments.

\section{Author contributions}

L.G., D.J.H. and M.J.S. conceived and designed the research. L.G., Z.Q., E.A. and J.J. performed the experiments. L.G., D.J.H., S.B.H., D.J.M. and M.J.S. analysed the data. L.G. and M.J.S. wrote the manuscript.

\section{Additional information}

Supplementary Information accompanies this paper at http://www.nature.com/ naturecommunications

Competing financial interests: The authors declare no competing financial interests.

Reprints and permission information is available online at http://npg.nature.com/ reprintsandpermissions/

How to cite this article: Gu, L. et al. In vivo time-gated fluorescence imaging with biodegradable luminescent porous silicon nanoparticles. Nat. Commun. 4:2326 doi: $10.1038 /$ ncomms3326 (2013). 